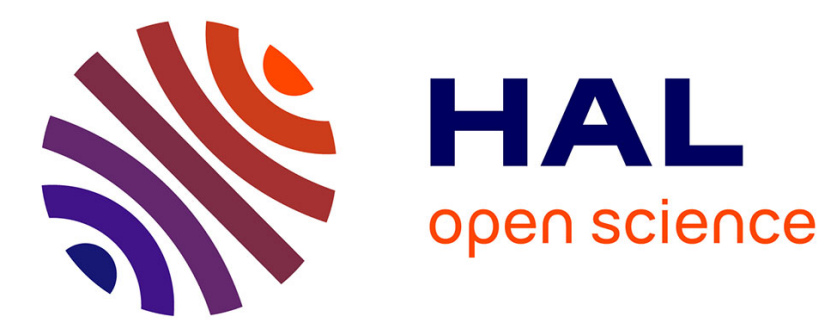

\title{
An efficient bit-loading algorithm with peak BER constraint for the band-extended PLC
}

Ali Maiga, Jean-Yves Baudais, Jean-François Hélard

\section{To cite this version:}

Ali Maiga, Jean-Yves Baudais, Jean-François Hélard. An efficient bit-loading algorithm with peak BER constraint for the band-extended PLC. ICT 2009, May 2009, Marrakech, Morocco. pp.292 - 297, 10.1109/ICTEL.2009.5158661. hal-00444414

\section{HAL Id: hal-00444414 https://hal.science/hal-00444414}

Submitted on 6 Jan 2010

HAL is a multi-disciplinary open access archive for the deposit and dissemination of scientific research documents, whether they are published or not. The documents may come from teaching and research institutions in France or abroad, or from public or private research centers.
L'archive ouverte pluridisciplinaire HAL, est destinée au dépôt et à la diffusion de documents scientifiques de niveau recherche, publiés ou non, émanant des établissements d'enseignement et de recherche français ou étrangers, des laboratoires publics ou privés. 


\title{
An Efficient Bit-Loading Algorithm With Peak BER Constraint For The Band-Extended PLC
}

\author{
Ali Maiga, Jean-Yves Baudais and Jean-François Hélard \\ Institute of Electronics and Telecommunications of Rennes \\ 35043 Rennes Cedex, France \\ Email: \{ali.maiga, jean-yves.baudais, jean-francois.helard\}@insa-rennes.fr
}

\begin{abstract}
Powerline communications (PLC) have become a viable local area network (LAN) solution for in-home networks. In order to achieve high bit rate over powerline, the current technology bandwidth is increased up to $100 \mathrm{MHz}$ within the European project OMEGA. In this paper, an efficient bit-loading algorithm with peak BER constraint is proposed. This algorithm tries to maximize the overall data rate based on linear precoded discrete multitone (LP-DMT), which enables reliable high bit rate transmission. A fast computational algorithm with mean BER constraint is also proposed. Simulations are run over PLC channels and it is shown that peak BER constraint algorithm combined with linear precoding component gives better performance than mean BER constraint algorithm.
\end{abstract}

\section{INTRODUCTION}

The FP7 OMEGA (Home Gigabit Networks) project is devoted to develop innovations in transmission technology and convergence layer for wireless i.e., LAN, ultra wide band (UWB), $60 \mathrm{GHz}$ systems, visible light communication systems and PLC systems [1], [2]. In our vision, the OMEGA home network will support Gbps transmission with low latency, and high coverage within the home, and then to the access network. The network concept is depicted in Fig. 1. Initially developed in the 1980's for low bit rate applications, such as telemetry, infrastructure control and consumption measurement, powerline communication (PLC) technologies have recently drawn an increasing interest within the scientific community for high bit rate communications over the power grid. Due to significant advances achieved in terms of modulation and signal processing schemes on one hand, and owing to the ubiquity of the outlet on the other hand, PLC is today considered as a solution of high potential for indoor applications and constitutes a convenient and cheap alternative to already existing technologies. Current systems use the spectral region up to $30 \mathrm{MHz}$. In OMEGA project, the possible higher bandwidth communications up to $100 \mathrm{MHz}$ is investigated.

The powerline channel exhibits multipaths caused by reflections on the discontinuities of the network and offers impulse responses that can be assumed as quasi-static. These two main features encourage the use of robust communication systems for the former and the exploitation of the knowledge of the channel state information (CSI) at the transmitter side for the latter. Under this assumption, adaptive bit-loading principles can advantageously be applied using discrete multitone modulation (DMT) in the PLC context [3]. Many loading

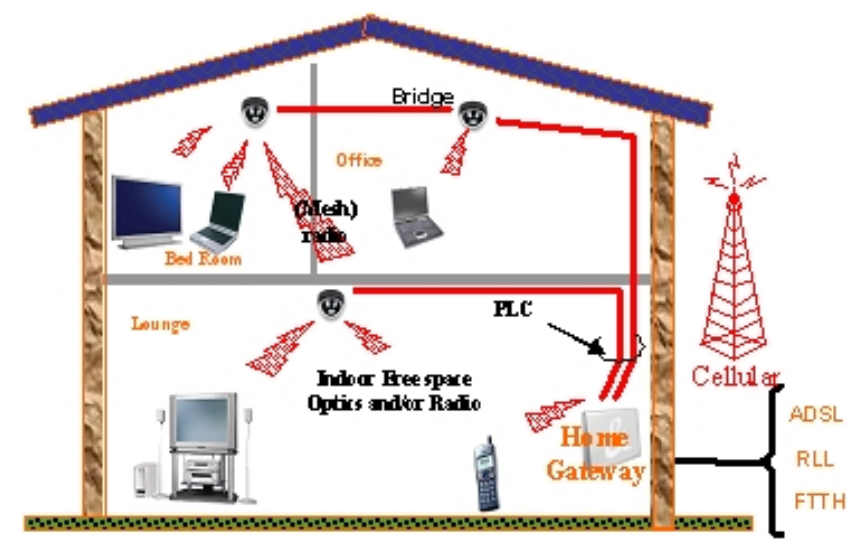

Fig. 1. Ultra-broadband home area network - OMEGA.

algorithms for allocating power and bits have been developed and are based on the well known waterfilling approach. The first proposed [4] algorithm for xDSL applications reaches the optimal solution using a greedy algorithm but leads to intensive computational complexity when the number of bits to be transmitted per DMT symbol is large. Many suboptimal algorithms with less computational complexity have since been developed (refer, for example, [5] and references inside for an extensive overview).

In [6], [7], the proposed bit-loading algorithms try to maximize the overall PLC throughput under a mean bit error rate (BER) constraint. Although these algorithms give good results, their computational complexity is still rather high at low SNR values. What is needed is an algorithm that accurately determines the final bit allocation in a low computational complexity fashion.

In this paper, an efficient bit-loading algorithm with peak BER constraint is proposed for the band-extended PLC. As in power constraint, a peak constraint is defined in opposition to average constraint [12]. In PLC context, the peak power constraint is the power spectral density (PSD) constraint where the power is limited for each subcarrier. In the case of BER, this constraint is applied to each bit. This new algorithm dynamically allocates subcarriers, bits and energies based on the linear precoding technique. It is much simpler to implement, since no convergence iterations are required, but simply a look-up table is used, in order to store, for each 
allowable modulation order, the required channel conditions to guarantee a target BER. Linear precoded DMT (LP-DMT) is a combination of multi-carrier and spread spectrum techniques also known as MC-SS techniques. This technique has shown very good performances in difficult environments and brings a significant increase in bit rate compared to classical DMT systems [15], [16]. For comparison purposes, a fast computational bit-loading with mean BER constraint is also proposed.

This paper is organized as follows. Section II describes the studied PLC systems. Section III presents the bit-loading algorithms with mean BER constraint and the proposed modified version. Section IV gives the new proposed bit-loading algorithm under peak BER constraint. The performance of the proposed algorithms over PLC channels is given in section V. Finally, section VI concludes the paper.

\section{SYSTEMS DESCRIPTION}

Homeplug AV (HPAV) has been standardized by Homeplug Powerline Alliance in order to improve the previous Homeplug 1.0 standard. While Homeplug 1.0 was designed to distribute the Internet access, HPAV aims at supporting Audio/Video as well as data traffic within home. HPAV employs the advanced PHY and MAC technologies and provides up to the PHY rate of $150 \mathrm{Mbps}$. It operates in the frequency range of 0 $37.5 \mathrm{MHz}$ with 1536 subcarriers and uses the same MAC protocol of CSMA/CA as Homeplug 1.0. In addition, to achieve signaling bit rates near theoretical capacity, HPAV specifications prescribe the use of turbo convolutional codes for forward error-correction coding [8]. Owing to electromagnetic compatibility constraint, a PSD mask is defined and only 917 usable subcarriers (tones) are used in order not to interfere with amateur radios [8], [9]. In addition, modulation densities from BPSK to 1024 QAM are independently applied to each subcarrier based on the link budget between the transmitter and the receiver. Consequently, all these limitations reduce significantly the total bit rate.

The band-extended PLC system studied within the European ICT FP7 OMEGA project will focus on developing a wide band (up to $100 \mathrm{MHz}$ ) transmission interface that allows for coexistence and compatibility with the existing HPAV specifications. Therefore, the same carrier spacing of $24.414 \mathrm{kHz}$ is kept as HPAV for this new system. There are 4096 carriers from DC to $100 \mathrm{MHz}$. The modulation densities are from BPSK to 32768 QAM (15 bits). Regarding Electromagnetic Compatibility (EMC), the electromagnetic emission limits for information technology equipment are specified as conducted emission limits in frequency range below $30 \mathrm{MHz}$ and as radiated emission for the frequencies above $30 \mathrm{MHz}$. A primary observation demonstrates that the PSD level in the 30$100 \mathrm{MHz}$ would be around $-80 \mathrm{dBm} / \mathrm{Hz}$ in order to respect the EMC constraints [10]. A PSD tone mask (Fig. 2) as in HPAV is performed, where 4 or 5 additional subcarriers on either side of the each notch are set to zero amplitude in order to guarantee that the energy inside the licensed band will be at least $30 \mathrm{~dB}$ lower than the normal transmit power [17].

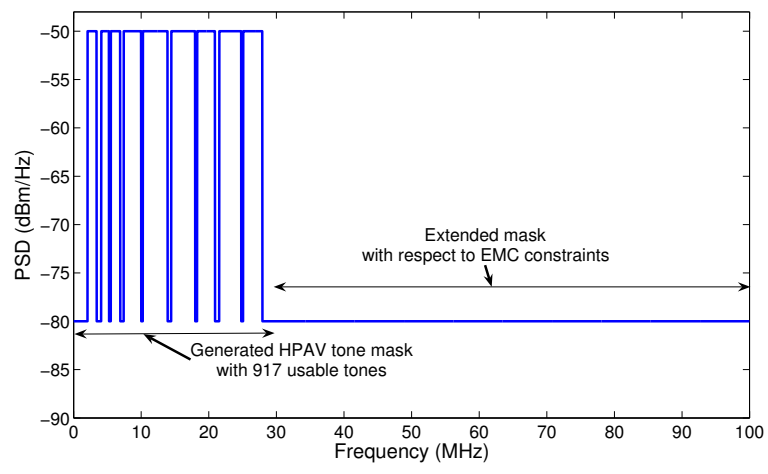

Fig. 2. Proposed band-extended PLC PSD mask.

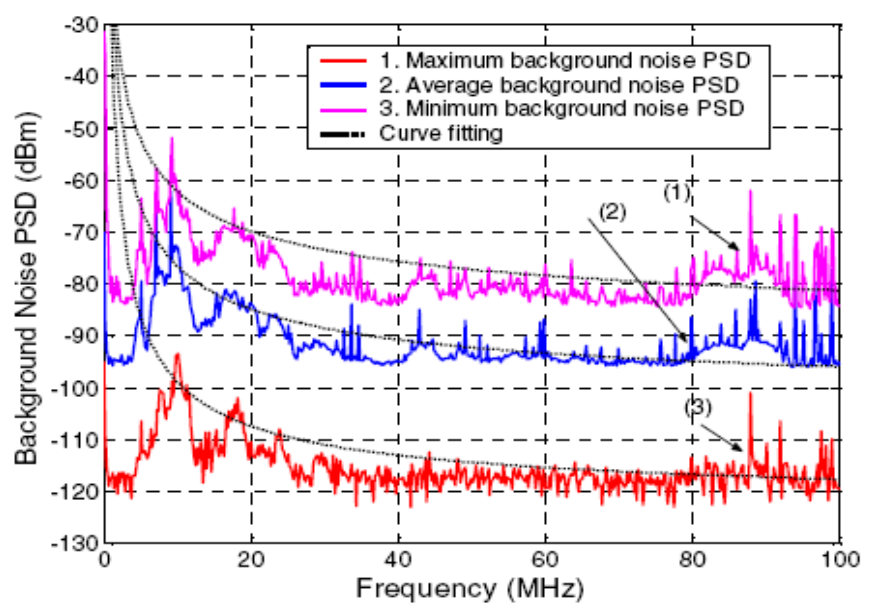

Fig. 3. Maximum, minimum and the average of colored background noise [13].

The powerline noise can be generally classified into four types: colored background noise, narrow-band noise, periodic impulse noise, random impulse noise. Narrow-band noise consists of sinusoidal signals with the modulated amplitudes caused by the ingress of broadcast stations. Periodic impulse noise is normally generated by appliances that produce harmonics at the frequencies of 50-100 Hz. Besides those, background noise, and asynchronous, or so-called random impulse noise, plays an important role in PLC communications [13]. In the following, only the background noise effect is taken into account. In [13], the power spectrum density expressed in $\mathrm{W} / \mathrm{Hz}$ is given by

$$
N_{0}(f)=10^{b-c . f^{d}} .
$$

The value of $b$ is a function of time and location. Based on measurements, $b$ is almost constant for long periods of time, and can be modeled to follow Gaussian distribution. $c$ is determined based on measurements, and the typical value of $d$ is $0.5-0.7$. The maximum, minimum and the average of colored background noise of equation (1) are shown in Fig. 3. 


\section{Mean BER CONSTRAint (MBC) BIT-LOADING ALGORITHM}

The term DMT denotes orthogonal frequency division multiplex (OFDM) based communication systems that adapt the transmission to the channel conditions individually for each subcarrier, by means of so called bit-loading. This technique is currently used for XDSL solutions and HPAV. The number of bits $b_{n}$ on each subcarrier $n$ depends on the signal-to-noise ratio $\mathrm{SNR}_{n}$ of the subcarrier $n$ and the BER constraint $\left(\mathrm{BER}_{n}\right)$ approximated by the normalized SNR $\Gamma_{n}$ (also known as SNR gap). The BER on subcarrier $n$ is approximated as

$$
\mathrm{BER}_{n}=\frac{4}{b_{n}} Q\left(\sqrt{3 \Gamma_{n}}\right)=\frac{4}{b_{n}} Q\left(\sqrt{3 \frac{\mathrm{SNR}_{n}}{\left(2^{b_{n}}-1\right)}}\right)
$$

The adaptive bit-loading algorithm with mean BER constraint (MBC) proposed in [6], [7], tries to solve the following problem

$$
\left\{\begin{array}{l}
\max _{b_{n}} \sum_{n=1}^{N} b_{n} \\
\overline{b e r}=\frac{\sum_{i=n}^{N} b_{n} \mathrm{BER}_{n}}{\sum_{n=1}^{N} b_{n}} \leq \widehat{b e r},
\end{array}\right.
$$

where $\overline{b e r}$ and $\widehat{b e r}$ are respectively the mean BER and the BER constraint. In [7], it is proposed an incremental bitloading algorithm based on a greedy approach to solve this problem. Although this incremental algorithm usually attains near-optimal solutions, its computational complexity is still rather high at low SNR values. What is needed is an algorithm that accurately determines the final bit allocation in an iterative low computational complexity fashion. In order to reduce the complexity, an algorithm which tries to estimate a new BER constraint for all subcarriers before doing the allocation is proposed in [6], [7]. Knowing the new BER constraint, the algorithm allocates the bits to subcarriers and reduces the bits allocated to subcarriers with worst BER until the mean BER constraint is satisfied. Our proposed algorithm tries to find the subcarriers that can use a greater BER while the mean BER constraint is satisfied. The algorithm is detailed in algorithm 1.

Apart from the iteration to find the largest value of $I$, the proposed algorithm do not need any more iterations. the computational time of this algorithm is compared with proposed algorithm in [6], [7] in table I. The computational times are given for several SNR values over Rayleigh fading channel with 4096 subcarriers under target BER of $10^{-5}$.

\section{A NEW PEAK BER CONSTRAINT (PBC) BIT-LOADING ALGORITHM}

As previously stated, LP-DMT results from the combination of multi-carrier modulation and spread spectrum. In our study, the LP component is not used to share access between users, as code division multiplex access (CDMA) does, but is used

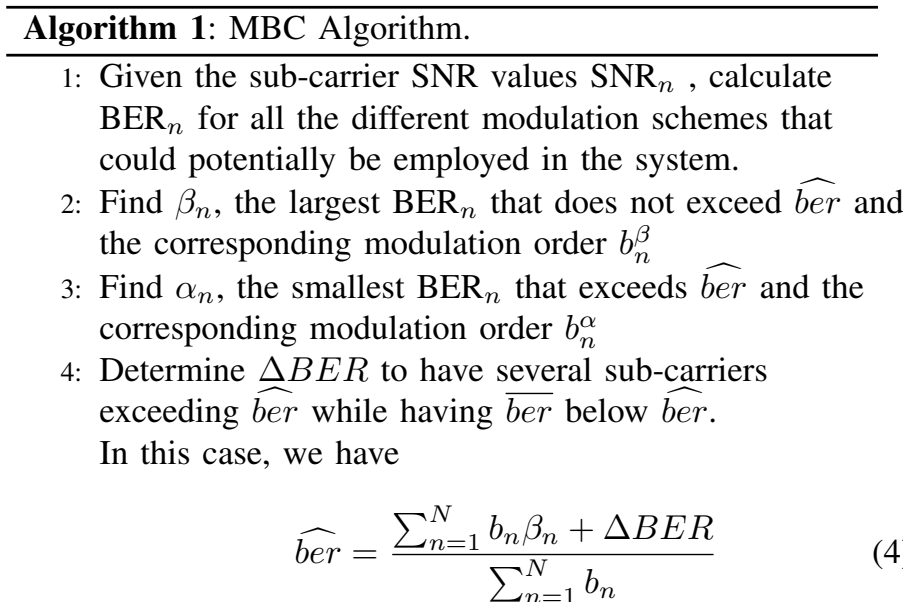

or equivalently

$$
\triangle B E R=\sum_{n=1}^{N} b_{n}\left(\widehat{b e r}-\beta_{n}\right) .
$$

We try to replace $\beta_{i}$ by $\alpha_{n}$ as far as it is possible until the mean BER constraint is satisfied.

We have, when assuming that $\alpha_{n}$ is sorted in increasing order, without loosing generality,

$$
\widehat{b e r} \geq \frac{\sum_{n=I+1}^{N} b_{n}^{\beta} \beta_{n}+\sum_{n=1}^{I} b_{n}^{\alpha} \alpha_{n}}{\sum_{n=I+1}^{N} b_{n}^{\beta}+\sum_{n=1}^{I} b_{n}^{\alpha}}
$$

and then,

$$
\Delta B E R+\left(\sum_{n=1}^{I}\left(b_{n}^{\alpha}-b_{n}^{\beta}\right)\right) \widehat{\operatorname{ber}} \geq \sum_{n=1}^{I}\left(b_{n}^{\alpha} \alpha_{n}-b_{n}^{\beta} \beta_{n}\right)
$$

5: Determine the largest value of $I$ for which (6) is true. Hence, the bit allocation becomes:

$$
b_{n}=\left\{\begin{array}{r}
b_{n}^{\alpha}(1 \leq n \leq I) \\
b_{n}^{\beta}(I<n \leq N)
\end{array}\right.
$$

\begin{tabular}{|c|c|c|c|}
\hline Algorithm & $20 \mathrm{~dB}$ & $35 \mathrm{~dB}$ & $50 \mathrm{~dB}$ \\
\hline Proposed in [6], & 1.9813 & 2.8375 & 2.7750 \\
[7] & $(2.2188)$ & $(3.1875)$ & $(3.1719)$ \\
\hline \multirow{2}{*}{ Our proposal } & 1.1453 & 1.4641 & 1.4719 \\
& $(1.2031)$ & $(1.5313)$ & $(1.5313)$ \\
\hline
\end{tabular}

TABLE I

MEAN (WORST CASE) COMPUTATION TIMES IN SECONDS AT DIFFERENT SNR VALUES USING MATLAB WITH INTEL CORE2@2.66GHz.

to multiplex different data symbols of a given user. The merging process consists in connecting a set of subcarriers with precoding sequences to mutually exploit their energies. This set of subcarriers is called block in the following and the subcarriers are not necessary adjacent. The number of blocks is defined as the ratio of total number of subcarriers $N$ to the precoding sequence length $L$. The classical DMT 
system is obtained for $L=1$. If judiciously done, each resulting set holds an equivalent SNR such that the total supported throughput is greater than the sum of the individual throughputs supported by each subcarrier taken separately. The linear precoding component is composed of Hadamard matrices.

When trying to maximize the bit rate under the peak BER constraint $\widehat{b e r}$, the optimization problem for a block $S$ of $L$ subcarriers in a single user case is given by [15], [16]

$$
\left\{\begin{array}{l}
\max _{C, E_{c}} R_{S}=\max _{C, E_{c}} \sum_{c=0}^{C-1} b_{c} \\
\text { with } b_{c}=\log _{2}\left(1+\frac{E_{c}}{\Gamma_{c} N_{0}} \frac{L^{2}}{\sum_{n \in S} \frac{1}{\left|H_{n}\right|^{2}}}\right) \\
\text { subject to, } \sum_{c=0}^{C-1} E_{c} \leq E \text { and } \frac{4}{b_{c}} Q\left(\sqrt{3 \Gamma_{c}}\right) \leq \widehat{b e r} .
\end{array}\right.
$$

The adaptive parameters are the energies $E_{c}$ allocated to each sequence and the number of precoding sequences $C$ within each block. There is an interdependency between the allocated bit $b_{c}$ to precoding sequence $c$ and the SNR gap $\Gamma_{c}$ given by

$$
\Gamma_{c}=\frac{E_{c} \times L^{2}}{N_{0}\left(2^{b_{c}}-1\right) \sum_{n \in S} \frac{1}{\left|H_{n}\right|^{2}}}=\frac{1}{3}\left(Q^{-1}\left(\frac{b_{c} \times \widehat{b e r}}{4}\right)\right)^{2},
$$

where $Q^{-1}$ is the inverse of the well-known Q-function given by

$$
Q(\alpha)=\frac{1}{\sqrt{2 \pi}} \int_{\alpha}^{+\infty} e^{-\frac{x^{2}}{2}} d x
$$

For a constant SNR gap $\Gamma$, over the block $S$ of $L$ subcarriers, it has been proven that the optimum achieved bit rate under PSD constraint is given by [15]

$$
R_{S}=L \times \log _{2}\left(1+\frac{E}{\Gamma N_{0}} \frac{L}{\sum_{n \in S} \frac{1}{\left|H_{n}\right|^{2}}}\right)
$$

where $E$ is the PSD constraint and $b_{c}=R_{S} / L$.

For a peak BER constraint (PBC), $\Gamma$ is no more constant for all modulation orders and bit-loading algorithms have to be performed with variable SNR gap $\Gamma_{c}$ for each modulation order $b_{c}$. In [11], it has been proposed bit-loading algorithms trying to solve this problem. The main idea in this algorithm is to iteratively allocate the bit to blocks of subcarriers until the satisfaction of peak BER constraint. In this paper, an efficient bit-loading algorithm with peak BER constraint is proposed for LP-DMT in a single user context. The main idea is to predefine the sum of inverse channel gains within a block $S$ which is needed to transmit $R_{S}$ bits. From (12), the needed sum of inverse channel gains (NSICG) is derived as

$$
\mathrm{NSICG}_{c}=\frac{E}{\Gamma_{b} N_{0}} \frac{L}{\left(2^{\dot{b_{c}}}-1\right)},
$$

when assuming the same discrete modulation order $\dot{b_{c}}$ for all subcarriers within the block $S$, and $\Gamma_{c}$ is computed using (10). Let $\dot{b_{c}}=\left\lfloor R_{S} / L\right\rfloor$ for the block $S$ of $L$ subcarriers, therefore

$$
\dot{b_{c}} \leq R_{S} / L<\dot{b_{c}}+1 \text {, }
$$

and then, as NSICG is a decreasing function of the modulation order $b_{c}$

$$
\frac{E}{\Gamma_{c} N_{0}} \frac{L}{\left(2^{b_{c}}-1\right)} \leq \frac{E}{\Gamma N_{0}} \frac{L}{\left(2^{\frac{R_{S}}{L}}-1\right)}<\frac{E}{\Gamma_{c+1} N_{0}} \frac{L}{\left(2^{b_{c}+1}-1\right)},
$$

where $\Gamma_{c+1} \leq \Gamma \leq \Gamma_{c}$. From (12), we derive

$$
\mathrm{NSICG}_{c} \leq \sum_{n \in S} \frac{1}{\left|H_{n}\right|^{2}}<\mathrm{NSICG}_{c+1} .
$$

Hence, for the block $S$, the achieved bit rate is computed from (12) setting $\Gamma=\Gamma_{c}$. For real systems, the bit rate achieved by an adaptive LP-DMT system using discrete modulation is maximized if, on block $S, \dot{b_{c}}$ bits are allocated to precoding sequence $c$, and $\dot{b_{c}}$ is given by [11]

$$
\dot{b_{c}}= \begin{cases}\left\lfloor R_{S} / L\right\rfloor+1 & \left(1 \leq i \leq n_{c}\right), \\ \left\lfloor R_{S} / L\right\rfloor & \left(n_{c}<i \leq L\right),\end{cases}
$$

where

$$
n_{c}=\left\lfloor\frac{L\left(2^{R_{S} / L}-2^{\left\lfloor R_{S} / L\right\rfloor}\right)}{\left(2^{\left\lfloor R_{S} / L\right\rfloor+1}-1\right) \frac{\Gamma_{c+1}}{\Gamma_{c}}-\left(2^{\left\lfloor R_{S} / L\right\rfloor}-1\right)}\right\rfloor .
$$

Then, the achievable bit rate $\dot{R_{S}}$, is given by

$$
\dot{R}_{S}=n_{c} \times\left(\left\lfloor R_{S} / L\right\rfloor+1\right)+\left(L-n_{c}\right) \times\left\lfloor R_{S} / L\right\rfloor
$$

Each precoding sequence energy contribution in the total energy of the given block, is given by

$$
E_{c}=\left(2^{\dot{b_{c}}}-1\right) \frac{\Gamma_{c} N_{0}}{L^{2}} \sum_{n \in S} \frac{1}{\left|H_{n}\right|^{2}}
$$

which satisfies

$$
\sum_{c} E_{c} \leq E
$$

The bit-loading algorithm for a block $S$ of $L$ subcarriers is given in algorithm 2 . This algorithm is much simpler to implement, since no convergence iterations are required, but simply a look-up table in order to store, for each allowable modulation order $b_{c}$, the required $\Gamma_{c}$ and $\mathrm{NSICG}_{c}$ to guarantee a target peak BER. For multi block systems, the aforementioned bitloading algorithm is applied for each block. Subcarriers that maximize the overall throughput are then chosen. It has been proven that choosing the best available subcarriers for each block maximizes the system throughput [15]. 


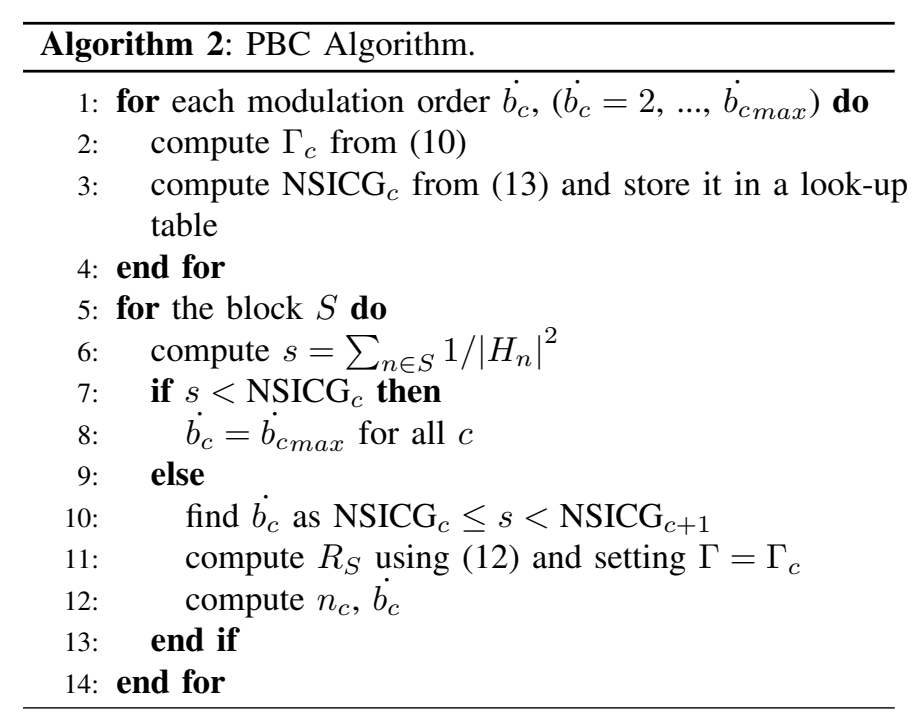

\section{Simulation Results}

In this section, simulation results for proposed resource allocation schemes are presented for the band-extended PLC systems. The generated signal is composed of $N=4096$ subcarriers transmitted in the band 0-100 MHz. Perfect synchronization and channel estimation are assumed. A high background noise level of $-110 \mathrm{dBm} / \mathrm{Hz}$ is assumed and the signal is transmitted with respect to a flat PSD of $-50 \mathrm{dBm} / \mathrm{Hz}$ and the PSD mask specified in Fig. 2 is directly applied to channel transfer function. Results are given for a fixed target BER of $10^{-3}$. For simplicity, the same precoding sequence length of 32 is assumed for all blocks of subcarriers and the maximum number of bits per symbol is limited to 15 . The multipath channel model given in [14] for indoor PLC is used. In [14], PLC channels are classified into 9 classes according to their capacities, and a model of transfer function is associated to each class. The higher the channel class number, the better its channel conditions. In the following, only three channel classes (class 2, 5 and 9) are considered and their transfer function models are given in table II.

\begin{tabular}{|c|c|}
\hline Class & Channel model \\
\hline \hline 2 & $-43+25 \times \exp \left(-\frac{f}{3.10^{6}}\right)-\frac{15}{10^{8}} f$ \\
\hline 5 & $-27+17 \times \exp \left(-\frac{f}{3.10^{6}}\right)-\frac{15}{10^{8}} f$ \\
\hline 9 & $-13+17 \times \cos \left(\frac{f}{4.5 .10^{7}}-0.5\right)$ \\
\hline
\end{tabular}

TABLE II

TRANSFER FUNCTION CHANNEL MODEL BY CLASS.

In a first step, different BER constraint algorithms are compared and first simulations are run over the class 5 channel. Fig. 4 depicts the achieved bit rates for different bit-loading

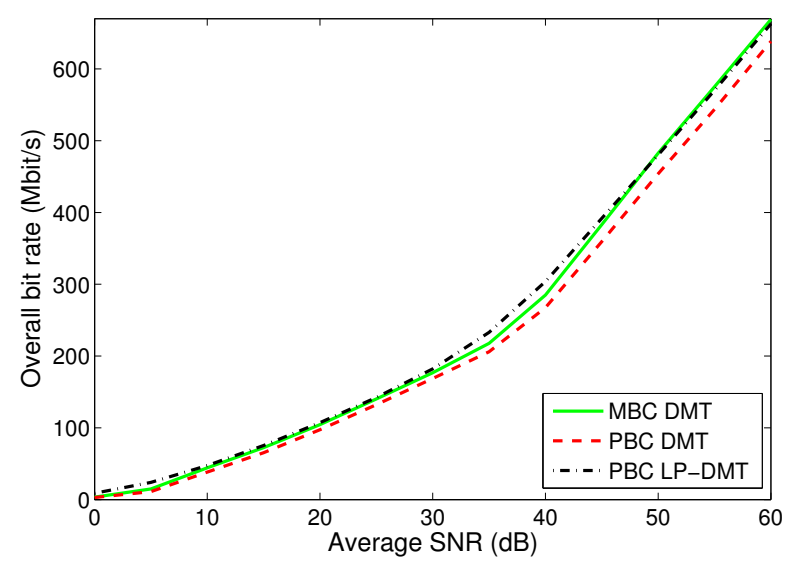

Fig. 4. Achieved bit rates for BER constraint for class 5 channel.

algorithms when the average SNR varies. The MBC-DMT and the PBC-DMT algorithms show the difference of mean BER and peak BER constraint algorithms. It is observed that bitloading with mean BER constraint (MBC-DMT) gives better results than bit-loading with peak BER constraint (PBC-DMT) in DMT context. The difference between the PCB-DMT and PCB-LP-DMT shows the bit rate gain brought by the linear precoding component. It shows that the linear precoding component bit rate gain is higher than gain brought by the mean BER constraint except for high SNR values $(\geq 50 \mathrm{~dB})$. For an average SNR value of $40 \mathrm{~dB}$, the PBC-LP-DMT algorithm with a precoding sequence length equal to 32 offers a bit rate of $303 \mathrm{Mbit} / \mathrm{s}$ that is a percentage increase of about 13\% compared to the PBC-DMT algorithm (267 Mbit/s). And the MBC-DMT algorithm offers a bit rate of $285 \mathrm{Mbit} / \mathrm{s}$ that is a percentage increase of about $6.7 \%$ compared to the PBC-DMT algorithm. The reason for the better performance of the LPDMT system is explained in Fig. 5, where the used energy of different peak BER constraint algorithms are compared. Fig. 5 gives the used energy per subcarrier for only 1024 subcarriers. To highlight the energy distribution, these subcarriers are sorted in descending order. The used energy is the minimal required energy allowing the transmission of the maximum data rate. Jump positions in curves correspond to the change in modulation orders. It is clear that the DMT algorithm (PBCDMT) is not fully exploiting the available energy on each subcarrier due to discrete modulation orders. The precoding component accumulates the residual lost energies of a given block of subcarriers to transmit additional bits. The adaptive LP-DMT system utilizes more efficiently the PSD limit.

In the second step, results are given for different channel classes and only three channel classes (class 2, 5 and 9) are considered. The bit-loading algorithms with BER constraint are performed for the band-extended PLC. Fig. 6 shows the achieved bit rate for class 2, 5 and 9 channels in single user context. This figure confirms what is highlighted previously. For all channel classes, the MBC algorithm outperforms the PBC-DMT algorithm. In addition, PBC-LP-DMT algorithm 


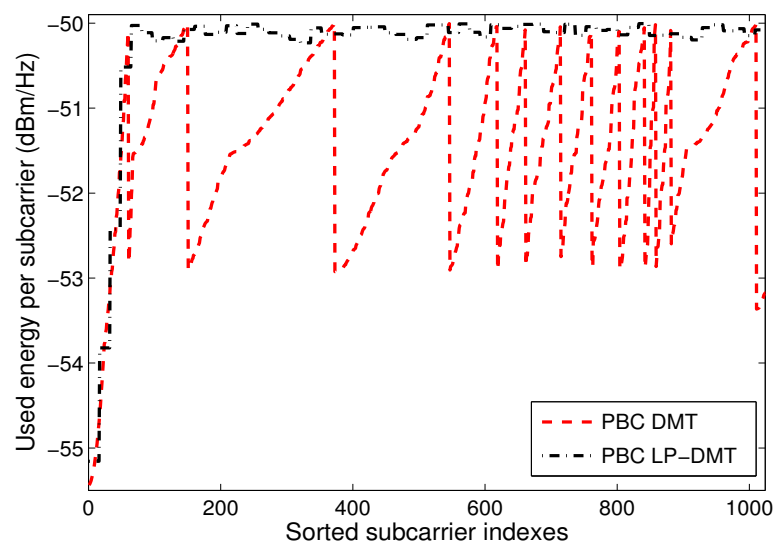

Fig. 5. Used energy for class 5 channel for $P B C$ algorithms at $\mathrm{SNR}=40 \mathrm{~dB}$.

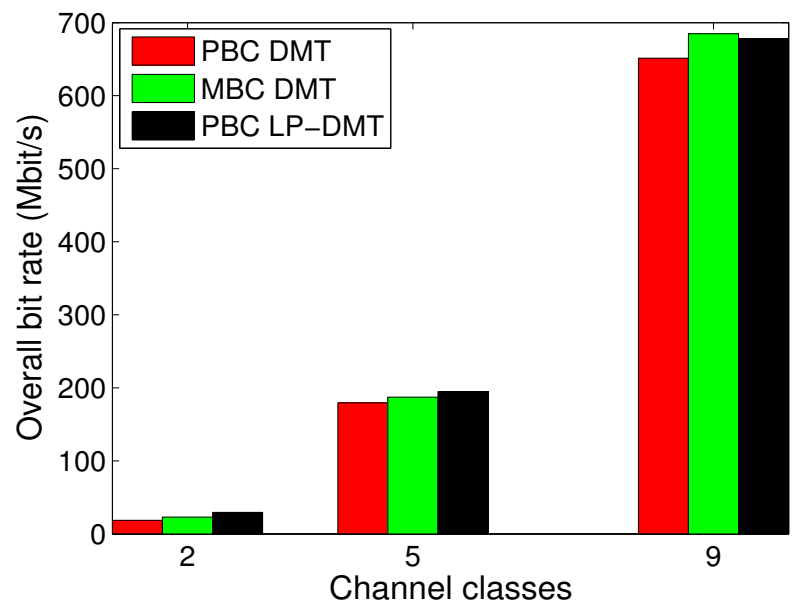

Fig. 6. Achieved bit rate for class 2, 5 and 9 channels.

gives better results than $\mathrm{MBC}$ algorithm apart from the best channel class 9, where $\mathrm{MBC}$ algorithm is the best.

However, the PBC algorithms remain much simpler to implement in PLC context, since no convergence iterations are required, but simply a look-up table is used, in order to store, for each allowable modulation order, the required channel conditions to guarantee a target peak BER.

\section{CONCLUSION}

In this paper, an efficient bit-loading algorithm with peak BER constraint has been proposed for the band-extended PLC system in home networks. For comparison purposes, a fast computationally-time version of mean BER constraint algorithm has been also proposed. It has been shown through simulations that the proposed peak BER constraint algorithm combined with linear precoding component gives better performances than mean BER constraint algorithm in PLC context.

\section{ACKNOWLEDGMENT}

The research leading to these results has received funding from the European Community's Seventh Framework Pro- gramme FP7/2007-2013 under grant agreement $n^{\circ} 213311$ also referred to as OMEGA.

\section{REFERENCES}

[1] ICT OMEGA project, http://www.ict-omega.eu

[2] J.P. Javaudin, M. Bellec, "Gigabit Home Networks," OMEGA ICT Project, proc. of ICT 2008.

[3] J. M. Cioffi, "A multicarrier primer," November 1991, ANSI Contribution T1E1.4/91-157, Clearfield, Fla, USA.

[4] D. Hughes-Hartogs, "Ensemble modem structure for imperfect transmission media," U.S. Patent 4679 227, July, 1987.

[5] S. Morosi, E. Del Re, R. Fantacci and D. Forasacchi, "Turbo-coding and bit-loading algorithms for a HomePlug-like DMT PLC system,' IEEE ISPLC 2006, pp. 227-231, March 2006.

[6] E. Guerrini, G. Dell'Amico, P. Bisaglia, L. Guerrieri, "Bit-loading algorithms and SNR estimate for HomePlug AV" IEEE ISPLC '07, pp.419-424, 26-28 March 2007

[7] A. M. Wyglinski, F. Labeau and P. Kabal, "Bit loading with BER constraint for multicarrier systems," IEEE Trans. on Wireless Comm., vol. 4, pp. 1383-1387, July 2005.

[8] "HomePlug AV white paper," http://www.homeplug.org.

[9] K. H. Afkhamie, S. Katar, L. Yonge and R. Newman, "An overview of the upcoming HomePlug AV standard” ISPLC 2005, pp. 400-404, April 2005.

[10] A. Zeddam, R. Razafferson, F. Gauthier and P. Pagani, "Analysis of EMC issues and throughputs of the PLC systems up to $100 \mathrm{MHz}$," UrsiGA 08, May 2008, Chicago USA.

[11] F.S. Muhammad, J.-Y. Baudais, J.-F. Hélard and M. Crussière, "Coded adaptive linear precoded discrete multitone over PLC channel," ISPLC'08, April 2008.

[12] N. Papandreou and T. Antonakopoulos, "Bit and Power Allocation in Constrained Multicarrier Systems: The Single-User Case," EURASIP JASP, vol 2008, 14 pages, 2008

[13] E. Liu, Y. Gao, G. Samdani, O. Mukhtar, and T.Korhonen, "Broadband characterization of indoor powerline channel and its capacity consideration," in IEEE International Conference on Communications, vol. 2, May 2005, pp. 901-905.

[14] M. Tlich, A. Zeddam, F. Moulin, F. Gauthier, and G. Avril,"A broadband Powerline Channel Generator,” IEEE ISPLC07, pp. 505-510, Pisa, Italy, March 26-28, 2007.

[15] M. Crussière, J.-Y. Baudais, and J.-F. Hélard, "Adaptive linear precoded DMT as an efficient resource allocation scheme for power-line communications," Proc. of IEEE GLOBECOM'06, San Francisco, USA, November 2006.

[16] M. Crussière, J.-Y. Baudais, and J.-F. Hélard, "Adaptive SpreadSprectrum Multicarrier Multiple-Access Over Wirelines," IEEE JSAC, Vol. 24, No. 7, July 2006.

[17] B. Mashburn, H. Latchman, T. VanderMey, L. Yonge, K. Tripathi,"Signal processing challenges in the design of the HomePlug AV powerline standard to ensure co-existence with HomePlug 1.0.," IWSPAWC 2005, 5-8 June 2005. 